\title{
Perfil dermatoglífico das atletas de voleibol feminino da Copa da Rainha da Espanha
}

\author{
Adriano ALBERTI* \\ Gracielle FIN* \\ Gabriela SARTORI ${ }^{*}$ \\ Leoberto Ricardo GRIGOLLO* \\ Juan Manuel Garcia MANSO** \\ David Rodriguez RUIZ \\ Miriam Esther Quiroga ESCUDERO** \\ Rudy J osé NODARI J UNIOR*
}

*Universidade do Oeste de Santa Catarina, Joaçaba, SC, Brasil. **Universidad de Las Palmas de Gran Canaria, Calle Juan de Quesada Espanha.

\section{Resumo}

Situado entre os cinco maiores esportes de prestígio no mundo, o voleibol é um esporte que exige potência, agilidade, bem como alta velocidade de seus atletas, principalmente quando praticado em alto nível de competição. Seguindo esse contexto, torna-se importante o surgimento de ferramentas que possam servir de subsidio para a orientação de novos talentos na modalidade em questão. A dermatoglifia surge como um método possível para a busca e orientação de atletas no desporto de alto rendimento. Desta forma, o objetivo do presente estudo foi comparar as impressões digitais de atletas femininas de alto rendimento de Voleibol da Copa da Rainha da Espanha e de um grupo controle composto por mulheres não atletas. A amostra do estudo foi composta por 162 indivíduos do sexo feminino divididos em dois grupos. 0 primeiro composto por 81 atletas profissionais de equipes femininas de voleibol, participantes da Copa da Rainha de Voleibol da Espanha, que foram comparadas ao Grupo Controle, composto por 81 mulheres não atletas. A análise dermatoglífica seguiu o protocolo proposto por Cummins e Midlo, por intermédio do Leitor Dermatoglífico ${ }^{\circledR}$ validado por Nodari. Os resultados encontrados demonstraram que na comparação das variáveis numéricas, a quantidade de linhas do dedo médio da mão direita (MDSOL3) é menor no grupo das atletas profissionais de voleibol, quando comparados ao grupo controle. Os resultados encontrados neste estudo demonstraram que o perfil dermatoglífico das atletas de Voleibol feminino de alto rendimento difere significativamente do perfil da população não atleta. Recomendam-se novos estudos com populações de amostra maiores, com grupos étnicos variados, e pertencentes a diferentes competições e níveis competitivos do voleibol.

Palavras-chave: Voleibol; Mulheres; Dermatoglifia; Alto Rendimento.

\section{Introdução}

De fácil acesso e baixo custo o voleibol rapidamente espalhou-se pelo mundo conquistando novos adeptos, tornando-se um esporte de grande destaque mundial ${ }^{1}$. Segundo a Federação Internacional de Voleibol (FIVB), este se encontra entre os cinco maiores esportes de prestígio no mundo, além de ser uma modalidade olímpica e possuir 220 federações nacionais filiadas ${ }^{2}$.

O voleibol é um esporte que exige potência, agilidade, bem como alta velocidade ${ }^{3}$. Programas de treinamento recomendados para a modalidade incluem aspectos da capacidade aeróbica, força, potência e agilidade ${ }^{4,5}$. Diante desta modalidade que exige cada vez mais de seus atletas, torna-se de fundamental importância o desenvolvimento de novos mecanismos que visem à melhora no desempenho dos atletas.

A busca por ferramentas que intentam aperfeiçoar a performance humana tem sido cada vez mais intensa no âmbito esportivo. $\mathrm{O}$ treinamento desportivo apresenta-se como um conjunto de atividades complexas que se organizam com o propósito de 
permitir um rendimento máximo, mesmo que por determinado período de tempo. Este rendimento depende do aperfeiçoamento desportivo, por meio da periodizaçáo de treinamento, da melhoria da técnica e da tática, além do controle psicológico do atleta ${ }^{6}$.

No entanto, a preparação física é apenas um dos elementos da equação que determina a condição de um atleta de elite. A composiçâao genética transmitida ao ser humano no momento de sua concepção é um fator determinante que deve ser considerado ${ }^{7}$. As Ciências do Esporte ainda apresentam dificuldades em encontrar metodologias apropriadas para a detecção do potencial genético do indivíduo ${ }^{8}$.

Um método possível para a análise do potencial genético e de desenvolvimento fetal é a dermatoglifia, já que as impressóes digitais são compreendidas como representaçôes dérmicas destas características ${ }^{9,10}$.

Os desenhos das papilas dérmicas são estabelecidos entre a $13^{\mathrm{a}}$ e $19^{\mathrm{a}}$ semanas de gestação, simultaneamente ao sistema nervoso central do estrato blastogênico e do ectoderma. As impressóes digitais não se alteram durante toda a vida, incluindo o tipo de desenho e a quantidade de linhas nos dedos das mãos e pés, palmas das mãos e dos pés, a complexidade concisa dos desenhos e a quantidade total de linhas ${ }^{11}$.

Estudos baseados na dermatoglifia estabeleceram que a complexidade dos desenhos das impressóes digitais poderia prognosticar determinadas características genotípicas de aptidão física ${ }^{12,13}$. As características dermatoglíficas poderiam então ser utilizadas no processo de avaliação do atleta, tanto no que se refere à performance de capacidades físicas quanto à compreensão morfofuncional do atleta.

Diversos estudos demonstraram que a preparação do atleta implica a utilização de um complexo de ferramentas necessárias para o alcance dos objetivos $^{14,15}$. Assim, o treinamento que por muito tempo fora baseado em tentativas e erros concede lugar a fundamentos mais objetivos resultantes de pesquisas científicas, em que são possíveis identificar métodos e ferramentas que contribuam para o processo de orientação e avaliação de atletas, visando seu melhor desempenho no alto rendimento. Com isso, demonstra-se a importância do estudo, que tem como objetivo comparar as impressóes digitais de atletas femininas de alto rendimento de Voleibol da Copa da Rainha da Espanha e de um grupo controle composto por mulheres não atletas.

\section{Método}

A amostra do estudo foi composta por 162 indivíduos do sexo feminino, divididos em dois grupos. O primeiro foi composto por 81 atletas profissionais de equipes femininas de voleibol que foram comparadas ao Grupo Controle, composto por 81 mulheres, observando a equidade de idade de ambos os grupos. As atletas de voleibol compunham equipes que disputaram a Copa da Rainha de Voleibol da Espanha no ano de 2011, a mais antiga competiçáo da modalidade e a segunda competição de maior significância na Espanha. As impressôes digitais foram coletadas na sede da competição em Albacete, Espanha, durante a disputa do certame.

A análise dermatoglífica seguiu o protocolo proposto por Cummins e Midlo', por intermédio do Leitor Dermatoglífico validado por NodARI ${ }^{16}$. Para a captura, processamento e análise de impressóes digitais foi utilizado um processo informatizado, ou seja, um leitor constituído de um scanner óptico de rolamento que coleta e interpreta a imagem e constrói um desenho, em código binário, que é capturado por software específico de tratamento e reconstrução de imagens reais em preto e branco. Sequencialmente a essa fase, a interferência do avaliador ocorre na marcação dos pontos núcleo e delta, quando, então, o software faz a identificação qualitativa da imagem e quantitativa de linhas, gerando a planilha informatizada resultante dos dados processados.

As análises estatísticas foram processadas no Statistical Package for the Social Science (SPSS), versão 20.0, em que foi estabelecido o nível de significância $\mathrm{p} \leq 0,05$. Quando comparados os dois grupos e as respectivas variáveis quantitativas para observar a distribuiçáo de normalidade, foi utilizado o teste de Kolmogorov-Smirnov. Quando da observação de distribuição não normal, aplicou-se para inferência o Teste Não Paramétrico Mann-Whitney na comparação entre variáveis numéricas, na mão esquerda, somatório da quantidade de linhas do dedo 1 polegar (MESQL1), mão esquerda, somatório da quantidade de linhas do dedo 2 - indicador (MESQL2), mão esquerda, somatório da 
quantidade de linhas do dedo 3 - dedo médio (MESQL3), mão esquerda, somatório da quantidade de linhas do dedo 4 - anular (MESQL4) e mão esquerda, somatório da quantidade de linhas do dedo 5 - mínimo (MESQL5); somatório da quantidade total de linhas da mão esquerda (SQTLE); mão direita, somatório da quantidade de linhas do dedo 1 - polegar (MDSQL1), mão direita, somatório da quantidade de linhas do dedo 2 - indicador (MDSQL2), mão direita, somatório da quantidade de linhas do dedo 3 - dedo médio (MDSQL3), mão direita, somatório da quantidade de linhas do dedo 4 - anular (MDSQL4) e mão direita, somatório da quantidade de linhas do dedo 5-mínimo (MDSQL5); somatório da quantidade total de linhas da mão direita (SQTLD); somatório da quantidade total de linhas - ambas as mãos (SQTL). Já o Teste T foi utilizado quando as

\section{Resultados}

Observada a distribuição não normal das variáveis quantitativas por meio do teste de Kolmogorov-Smirnov, foi realizado o teste não paramétrico Mann-Whitney, para comparação das variáveis numéricas e o teste paramétrico Teste $\mathrm{T}$, quando as variáveis se apresentarem normais. variáveis apresentaram-se de maneira normal.

Para a comparação de variáveis categóricas Arco (A), Presilha Radial (LR), Presilha Ulnar (LU), Verticilo (W), desenho da mão esquerda, dedo 1 (MET1), dedo 2 (MET2), dedo 3 (MET3), dedo 4 (MET4) e dedo 5 (MET5) e da mão direita, dedo 1 (MDT1), dedo 2 (MDT2), dedo 3 (MDT3), dedo 4 (MDT4) e dedo 5 (MDT5) foi utilizado o teste Qui-quadrado quando encontradas diferenças significantes.

A pesquisa foi aprovada com protocolo número 292.868 do Comitê de Ética em Pesquisa em Seres Humanos da Unoesc/HUST, de acordo com os padróes éticos de normas e diretrizes regulamentadoras da pesquisa envolvendo seres humanos, em conformidade com a Resolução n. 196/96 do Conselho Nacional de Saúde e com a Declaração de Helsinki.
O resultado encontrado demonstra que há diferença na quantidade de linhas no dedo médio da mão direita (MDSQL3), é menor no grupo das atletas profissionais de voleibol, quando comparado ao grupo controle, conforme observado na TABELA 1.

TABELA 1- Quantidade de linhas das impressões digitais dos dedos da mão esquerda (SQTLE), direita (SQTLD), total de ambas as mãos (SQTL) e diferença significativa encontrada entre o grupo de atletas profissionais de voleibol comparado ao grupo controle.

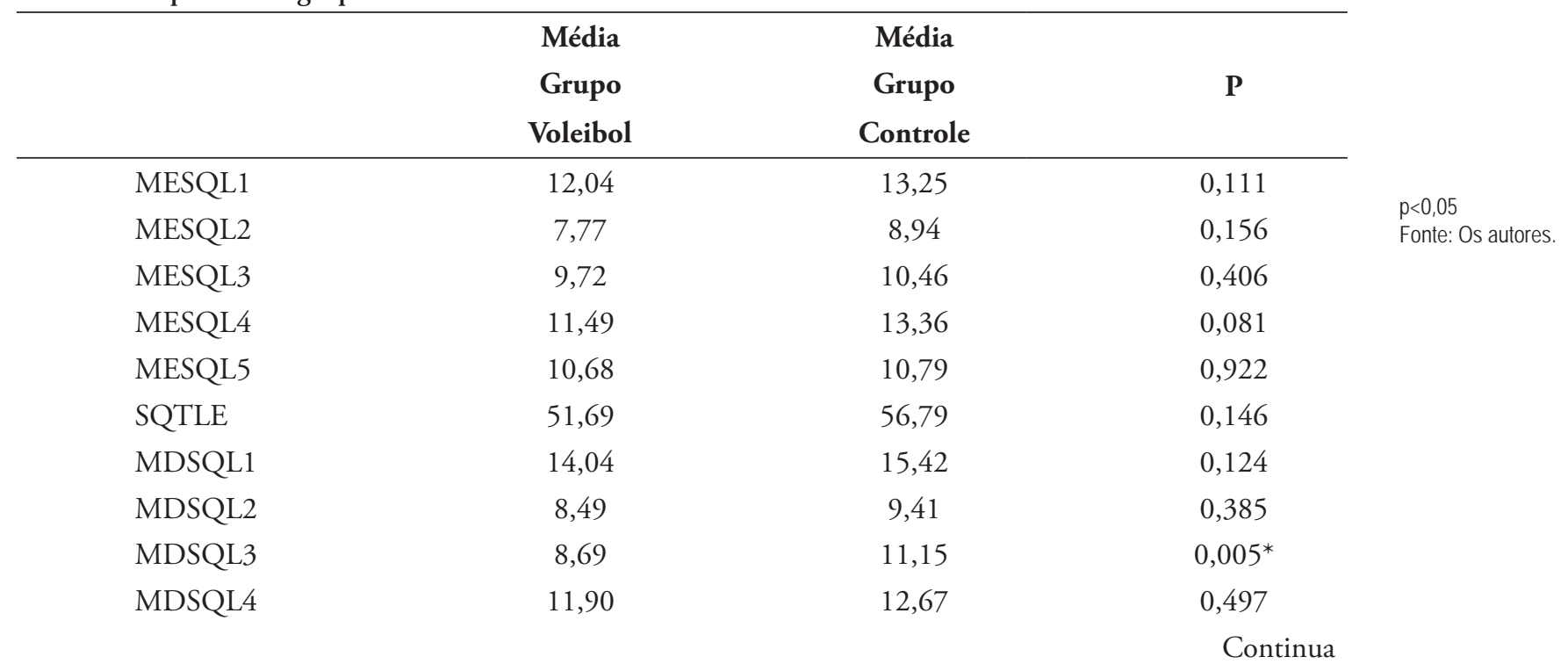


Continuação

$\begin{array}{lrrr}\text { MDSQL5 } & 10,91 & 11,16 & 0,912 \\ \text { SQTLD } & 54,04 & 59,80 & 0,156 \\ \text { SQTL } & 105,73 & 116,59 & 0,107\end{array}$

Quanto às variáveis categóricas o resultado do teste Qui-quadrado não demonstrou diferenças significativas entre os desenhos de $\operatorname{arcos}(\mathrm{A})$, presilha radial (LR), presilha ulnar (LU), verticilo (W) e S desenho, quando comparados os grupos de atletas profissionais com o grupo controle, entre os grupos analisados, conforme observado na TABELA 2.

TABELA 2 - Diferença significativa entre as figuras dos dedos da mão esquerda e direita encontrada quando comparados os grupos de atletas profissionais de voleibol ao grupo controle.

\begin{tabular}{cccccccccc}
\hline MET1 & MET2 & MET3 & MET4 & MET5 & MDT1 & MDT2 & MDT3 & MDT4 & MDT5 \\
0,760 & 0,186 & 0,574 & 0,665 & 0,866 & 0,433 & 0,289 & 0,496 & 0,914 & 0,328 \\
\hline
\end{tabular}

\section{Discussão}

A pesquisa demonstrou diferenças significativas nas atletas de alto rendimento de voleibol, quando comparadas a um grupo controle formado por mulheres não atletas, quanto às características dermatoglíficas quantitativas, ou seja, menor quantidade de linhas nas atletas investigadas no dedo médio da mão direita (MDSQL3), com a média de 8 linhas nas atletas e 11 nas náo atletas.

Em outra pesquisa feita com uma amostra de 12 indivíduos atletas de voleibol e sem a presença de um grupo controle, foi observado um comportamento de aumento da quantidade de linhas, mas também de diminuição ${ }^{17}$. Estes dados também foram observados em um estudo realizado apenas com atletas da seleção brasileira feminina de voleibol, em que o somatório da quantidade de linhas também apresentou resultado significativo, em que se destacou o maior número de linhas no dedo 1 da mão direita em relação à esquerda ${ }^{18}$. Porém, vale ressaltar que ambos estudos foram realizados sem um grupo controle, dando menos fidedignidade a pesquisa.

Em uma pesquisa realizada com atletas do sexo feminino com idade de 15 anos, convocadas para a seleção brasileira infantojuvenil de voleibol do Brasil, os autores ${ }^{19}$ verificaram a existência de diferença no somatório de linhas no SQTL, em posiçóes específicas dos jogadores no voleibol, de meio de rede, líbero e opostos. Entretanto, trata- se de uma amostra limitada a 19 atletas. Em um estudo realizado com atletas femininas do voleibol brasileiro, apresentou-se resultados no somatório de linhas não simétrico ${ }^{18}$. Assim como no estudo anterior, contempla uma pequena amostra, com apenas 28 atletas brasileiras, não sendo tão eficaz, mas tem grande influência no resultado obtido, não possuindo grupo controle para comparação.

Em outro estudo foram obtidos valores diferenciados nos grupos pesquisados quando avaliados atletas do sexo masculino da seleção brasileira de voleibol, categorias juvenil, infantojuvenil e adulto ${ }^{20}$. Os resultados revelaram altos valores no somatório total de linhas - SQTL em todos os grupos. Mas os atletas da categoria adulta obtiveram o maior valor, demonstrando diferença. $\mathrm{O}$ mesmo estudo não apresentou diferenças significativas nas demais variáveis. Assim como nos estudos anteriores ${ }^{19,20}$. utilizou-se do método de coleta com tinta e papel, porém náo é táo preciso quanto o Leitor Dermatoglífico ${ }^{\oplus}$ validado por Nodari ${ }^{16}$, além de limitar-se a uma pequena amostra de atletas.

Em outro estudo realizado com atletas de voleibol de alta qualificação e atletas de qualificação intermediária, verificou-se uma diferença significativa na quantidade de linhas, no qual os atletas de alta qualificação apresentaram um maior número de linhas em todos os dedos das duas máos, exceto 
MESQL5. No mesmo estudo verificou-se que os tletas de alta qualificaçáo apresentaram uma maior predominância de Verticilo $(\mathrm{W})^{21}$.

O diferencial deste estudo é a existência do grupo controle, pois possibilita a análise correlacional entre os grupos, evidenciando as diferenças encontradas, além da utilização do método informatizado desenvolvido e validado por Nodari ${ }^{16}$.

Em estudos realizados em outras modalidades de esportes de quadra, pode-se perceber valores diferenciados entre os grupos pesquisados ${ }^{8}$. Vale ressaltar que este estudo feito com atletas de basquetebol apresentou um comportamento diferenciado entre o grupo de atletas em comparaçáo com o grupo de não atletas, respectivamente no valor total da soma das linhas de todos os dedos-TRC (total ridge count).

Num estudo realizado na modalidade de futsal, com atletas do sexo masculino, Dantas, Alonso e FERNANDES ${ }^{22}$ verificaram altos índices no somatório total de linhas (SQTL). A amostra limitou-se a apenas atletas e foi feito um estudo comparativo em uma amostra anterior, na qual também se constatou resultados similares das duas amostras. Em outro estudo da modalidade futsal, porém com o sexo feminino, realizado por Alberti et al. ${ }^{23}$, os autores verificaram também altos índices no somatório total de linhas (SQTL), além da presença da figura Presilha Radial (LR) e Verticilo (W). Vale ressaltar que tal estudo ${ }^{23}$ foi o único realizado com o método informatizado ${ }^{24}$.

As contradiçóes entre os achados sobre dermatoglifia podem estar associadas aos diferentes métodos utilizados para as análises de impressóes digitais. Os resultados no presente estudo apresentam um diferencial para a dermatoglifia na modalidade de voleibol para o sexo feminino, uma vez que substitui a utilização do método tinta, papel e lupa da coleta tradicional de dermatoglifia pelo método informatizado ${ }^{24}$.

Sendo assim, podemos concluir que os grupos pesquisados apresentaram diferença significativa na quantidade de linhas do dedo 3 da máo direita (MDSQL3), demonstrando um perfil dermatoglífico distinto entre atletas de voleibol e grupo controle.

Os resultados apresentados neste estudo apontam que a dermatoglifia contribui na orientação de talentos e no desempenho do esporte de alto rendimento, sendo um método que vem a potencializar a avaliação de pessoas que demonstrem talento, permitindo identificar características genéticas e de desenvolvimento fetal, peculiares aos atletas. Com base nessas premissas, conta-se com mais uma ferramenta para auxiliar na busca de talentos no esporte, na qualificação do treinamento e do desempenho.

$\mathrm{Na}$ modalidade de Voleibol, torna-se de maior relevância mais estudos, pois ficou evidenciado diferenças dermatoglíficas importantes entre os grupos estudados. Por isso, recomenda-se que estudos futuros sejam realizados com populaçóes de amostra maiores, com grupos étnicos variados e pertencentes a diferentes competiçóes e níveis competitivos do voleibol.

\title{
Conflito de interesse
}

Informamos que não há conflito de interesse.

\begin{abstract}
Dermatoglyphic profile of female volleyball athletes of the Spanish Queen Cup

Classified among the five greatest prestigious sports in the world, volleyball requires power, agility and high speed from its athletes, mainly when it is practiced in high levels of competition. Following this context, it is important to have new tools arising that can provide support for the orientation of new talents in this modality. Dermatoglyphics comes as a feasible method to the pursuit and orientation of athletes in high-performance sports. Therefore, the objective of this study was to compare the fingerprints of high performance athletes of female volleyball of the Spanish Volleyball Queen Cup and a group control composed of non-athlete women. The sample of the study was composed of 162 female individuals divided into two groups. The first one was composed of 81 professional athletes of female volleyball teams participating of
\end{abstract}


the Spanish Volleyball Queen Cup, who were compared to the Control Group, composed of 81 women as well. The dermatoglyphic analysis followed the protocol proposed by Communs and Midlo' through the Dermatoglyphic Reader ${ }^{\circledR}$ validaded by Nodari ${ }^{16}$. The results obtained showed that in the comparison of the numerical variables, the total ridge count of right finger 3 (TRCRF3) is smaller in the group of volleyball professional athletes when compared to the control group. The results of the research showed that the dermatoglyphic profile of high performance athletes of female volleyball differ significantly from the profile of the non-athlete population. New studies are recommended with greater sample population, varied ethnic groups, and belonging to different competitions and competitive levels of volleyball.

KEYWORDS: Volleyball; Women; Dermatoglyphics; High performance.

\section{Referências}

1. Peres MM, Cecchini L, Pacheco IMPA. Efeitos do treinamento proprioceptivo na estabilidade do tornozelo em atletas de voleibol. Rev Bras Med Esporte. 2014;20:146-50.

2. Fédération Internationale de Volleyball. Volleyball history: the origins. 2016.

3. Taware GB, Bhutkar MV, Surdi AD. A profile of fitness parameters and performance of volleyball players. Jkimsu. 2013; 2(2):48-59.

4. Gonzalez-Rave JM, Arija A, Clemente-Suarez V. Seasonal changes in jump performance and body composition in women volleyball players. J Strength Cond Res. 2011;26:1492-501.

5. Rousanoglou EN, Barzouka KG, Boudolos KD. Seasonal changes of jumping performance and knee muscles strength in under -19 women volleyball players. J Strength Cond Res. 2013;27:1108-17.

6. Dantas EHM. Periodização do treinamento. A prática da preparação física. 5. ed. Rio de Janeiro: Shape, 2003.

7. Bouchard C, Hoffman EP. Genetic and molecular aspects of sport performance. Volume XVIII of the Encyclopaediaos Sports Medicine and IOC Medical Commission Publication. 1. ed. International Olympic Committee Published by Blackwell Publishing Ltd 2011.

8. Borin JP, Padovani CR, Araon FF, Gonçalves A. Dermatoglyphics in Sports Sciences: Understanding the distribution of quantitative indicators in non-athletes and athletes of basketball according to their performance. Rev Andal Med Deporte 2012;5(3):99-104.

9. Cummins H, Midlo CH. Finger Prints, Palms and Soles an Introduction to Dermatoglyphics. New York: Dover Publications; 1961.

10. Nodari RJ Jr, Fin G. Dermatoglifia: Impressóes digitais como marca genética e de desenvolvimento fetal. Joaçaba: Ed. Unoesc; 2016. Disponível em http://www.unoesc.edu.br/editora/livros-single/e-book-gratuito-dermatoglifia-impressoes-digitais-comomarca-genetica-e-de [2016 mar. 15].

11. Gonçalves A, Gonçalves NNS. Dermatóglifos: principais conceituaçóes e aplicações. An Bras Dermatol. 1985;59(4):173-186.

12. Nikitiuk BA. Impressões Dermatoglíficas como marcas do desenvolvimento pré-natal do ectoderma. Anais de trabalhos Científicos do Simpósio. Rimelnintski; 1988.

13. Guba VP, Tchernova GP. Meios morfo-biomecânicos baseados na determinação dos talentos e dotes esportivos. Organização da metodologia cientifica baseada nos sistemas de preparação dos atletas reservas russos e os caminhos do seu aperfeiçoamento. Anais do XV Congresso Científico da Rússia. 1995;115-6.

14. Zakharov A, Gomes A. Ciência do treinamento desportivo. Rio de Janeiro: Grupo Palestra Sport; 1992.

15. Volkov VM, Filin VP. Seleção Desportiva. Moscou: Fizcultura y Sport; 1983.

16. Nodari RJ Jr. Protótipo de escaneamento informatizado: possibilidade em diagnóstico em saúde por meio das impressóes digitais. [Tese de Doutorado]. Natal: Universidade Federal do Rio Grande do Norte; 2009.

17. Silva HG, Costa CLA, Silva HM, Capistrano RDS. Perfil dermatoglífico e qualidades físicas de jovens atletas de voleibol. Conexôes: Educ Fís Esporte e Saúde. 2010;8(1):1-15.

18. Fonseca CLT, Dantas PMS, Fernandes PR, Fernandes Filho J. Perfil dermatoglífico, somatotípico e da força explosiva de atletas da seleção brasileira de voleibol feminino. Fit Perf J. 2008;7(1):35-40.

19. Cabral SAT, Barbosa FP, Cabral BGAT, Knackfuss MI, Medeiros HJ, Fernandes Filho J. A seleção brasileira de voleibol infanto-juvenil feminina e o seu perfil dermatoglífico. Acta Cir Bras. 2005;20(1):22-6. 
20. Zary JCF, Fernandes Filho J. Identificação do Perfil dermatoglífico e somatotípico dos atletas de voleibol Masculino adulto, Juvenil e infanto-Juvenil , de alto Rendimento no Brasil. Rev Bras Ciênc Mov. 2007;15(1):53-60.

21. Fonseca C, Fernandes Filho J. Características Dermatoglíficas de Atletas de Voleibol Masculino Sub 17 do Brasil em Diferentes Níveis Qualificaçóes Esportiva. REAFES 2010; 122-9.

22. Dantas PMS, Alonso L, Fernandes Filho J. A dermatoglifia no futsal brasileiro de alto rendimento. Fit PerfJ. 2004; 3(3): 136-42.

23. Alberti A, Fin G, Vale RGS, Soares BH, Nodari RJ Jr. Dermatoglifia as impressóes digit como marca caracter dos atletas futsal fem alto rend do Brasil. Rev Bras Futsal e Futebol. 2018; 10(37):193-201.

24. Nodari RJ Jr., Heberle A, Ferreira-Emygdio R, Irany-Knackfuss M. Impressões Digitais para Diagnóstico em Saúde: Validação de Protótipo de Escaneamento Informatizado. Rev Salud Pública. 2008;10(5):767-76.

ENDEREÇO

Adriano Alberti

Universidade do Oeste de Santa Catarina

Rua J osé Firmo Bernardi, 1591, Flor da Serra

89600-000 - J oaçaba - SC - Brasil

E-mails: adrianoalberti90@hotmail.com adrianoalberti90@yahoo.com
Recebido para publicação: 15/04/2018

Revisado: 17/ 07/2018

Aceito: 15/11/ 2018 